\title{
Nonepitaxial Thin-Film InP for Scalable and Efficient Photocathodes
}

\author{
Mark Hettick, ${ }^{\dagger, \perp}$ Maxwell Zheng, ${ }^{\dagger, \dagger}$ Yongjing Lin, ${ }^{\dagger, \perp}$ Carolin M. Sutter-Fella, ${ }^{\dagger, \ddagger}$ Joel W. Ager, ${ }^{\perp, \ddagger}$ \\ and Ali Javey*,,,,++ \\ ${ }^{\dagger} E$ lectrical Engineering and Computer Sciences, University of California, Berkeley, California 94720, United States \\ ${ }^{\perp}$ Joint Center for Artificial Photosynthesis and ${ }^{\ddagger}$ Materials Sciences Division, Lawrence Berkeley National Laboratory, Berkeley, \\ California 94720, United States
}

\section{Supporting Information}

ABSTRACT: To date, some of the highest performance photocathodes of a photoelectrochemical (PEC) cell have been shown with single-crystalline p-type InP wafers, exhibiting half-cell solar-to-hydrogen conversion efficiencies of over $14 \%$. However, the high cost of single-crystalline InP wafers may present a challenge for future large-scale industrial deployment. Analogous to solar cells, a thin-film approach could address the cost challenges by utilizing the benefits of the $\mathrm{InP}$ material while decreasing the use of expensive materials and processes. Here, we demonstrate this approach, using the newly developed thin-film vapor-liquid-solid (TF-VLS) nonepitaxial growth method combined with an atomic-layer deposition protection process to create thin-film $\mathrm{InP}$ photocathodes with large grain size and high performance, in the first reported solar device configuration generated by materials grown with this technique. Current-voltage measurements show a photocurrent $(29.4 \mathrm{~mA} /$

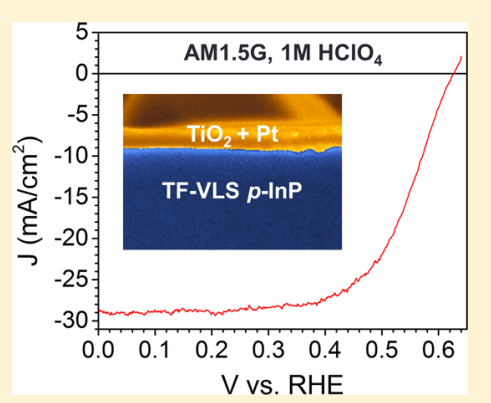
$\left.\mathrm{cm}^{2}\right)$ and onset potential $(630 \mathrm{mV})$ approaching single-crystalline wafers and an overall power conversion efficiency of $11.6 \%$, making TF-VLS InP a promising photocathode for scalable and efficient solar hydrogen generation.

$\mathrm{T}$ he generation of storable fuel from sunlight in the manner of photosynthesis is a promising solution to the clean energy problem, and the prospect of highly efficient photoelectrochemical (PEC) cells is an attractive route to generation of solar fuels. ${ }^{1-3}$ Due to the $1.23 \mathrm{eV}$ of free energy required for the water splitting process, in addition to the overpotentials generated at each electrode under operation, work on the tandem cell architecture with a particular focus on producing high-voltage half-cells is desirable to meet this requirement while maintaining adequate absorption across the solar spectrum. ${ }^{4}$ For the $\mathrm{H}_{2}$-producing photocathode half-cell, a variety of semiconductors have been explored, including planar, ${ }^{5-7}$ micro-, ${ }^{8}$ and nanostructured ${ }^{9}$ crystalline $\mathrm{Si}$, amorphous $\mathrm{Si}^{10}{ }^{10} \mathrm{InP},{ }^{11-17} \mathrm{WSe}_{2},{ }^{18} \mathrm{TiO}_{2}$-coated $\mathrm{Cu}_{2} \mathrm{O},{ }^{19,20}$ and $\mathrm{CdS}$ modified $p-\mathrm{CuGaSe}_{2}{ }^{21}$

In particular, p-type InP stands out for its direct band gap of $\sim 1.35 \mathrm{eV}$, suitable conduction band position in relation to the proton reduction potential, and associated high efficiency for the hydrogen evolution reaction. ${ }^{12-16}$ In fact, nanostructured crystalline InP wafers have achieved a half-cell solar-tohydrogen (STH) efficiency of approximately $14 \%,{ }^{12}$ which is the highest reported to date. In addition, other work on $\mathrm{TiO}_{2}$ passivated crystalline InP wafers resulted in half-cells with an onset potential greater than $800 \mathrm{mV}$, providing some of the high voltage necessary for unassisted water splitting in the ideal tandem cell configuration. ${ }^{13}$ Still, despite its high efficiency, prior work on InP as a PEC photocathode material has primarily focused on single-crystal wafers, which limits largescale application due to the high cost of the bulk substrates. In this work, we explore a more practical approach, utilizing a new high quality thin-film InP growth method ${ }^{22}$ on low-cost Mo substrates along with previously explored protection schemes ${ }^{7,10,12,13}$ to establish a low-cost and high-efficiency PEC system. The system, the first solar device structure implemented using the thin-film vapor-liquid-solid (TF-VLS) growth method, exhibits decreased overall material usage, high performance, and potential expansion to device structures requiring fine nucleation and growth control. ${ }^{22,23}$ Material and device quality is evaluated using imaging, photoluminescence (PL), and Mott-Schottky capacitance-voltage measurements, and performance of our photocathodes is evaluated through current voltage $(J-V)$ measurements to be compared with previous photocathode reports.

A full process scheme for our photocathodes is visualized in Figure 1. The TF-VLS growth method is chosen as it produces films with high optical and crystal quality with theoretical performance approaching that of the bulk crystal in terms of the luminescence efficiency and optically implied open-circuit voltage. ${ }^{22}$ Briefly, the TF-VLS growth method begins with an indium film approximately $2 \mu \mathrm{m}$ in thickness deposited on a molybdenum substrate, capped by a thin silicon oxide $\left(\mathrm{SiO}_{x}\right)$ layer (details are given in the Experimental Methods section). This stack is heated in hydrogen past the film's melting point, followed by the introduction of $\mathrm{PH}_{3}$ gas for phosphorization of the liquid indium by a vapor-liquid-solid growth mode. Importantly, the $\mathrm{SiO}_{x}$ layer confines the indium during growth to a planar thin-film geometry, allowing growth to proceed only

Received: April 9, 2015

Accepted: May 25, 2015 
(a)

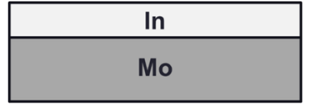

(d)
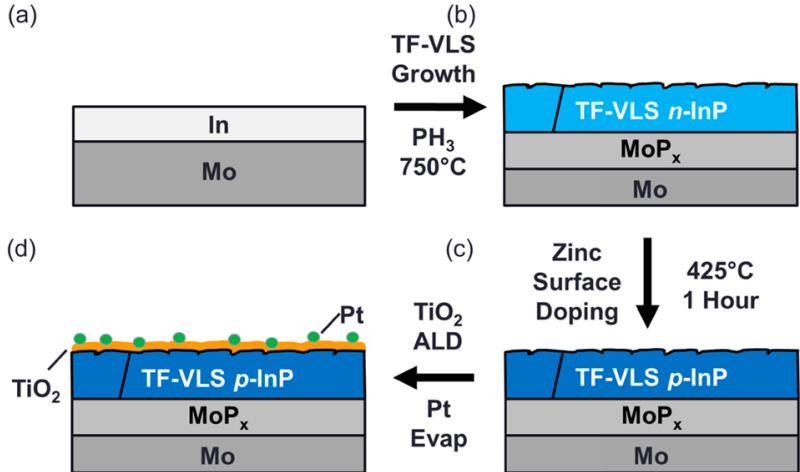

Figure 1. Fabrication process for TF-VLS InP PEC devices. (a) Indium metal is evaporated on the substrate using an electron beam evaporator. (b) An n-type (unintentionally doped) polycrystalline InP film is grown on the Mo substrate through a TF-VLS process. (c) The $\mathrm{n}$-type film is $\mathrm{p}$-doped by surface diffusion using a $\mathrm{Zn}_{3} \mathrm{P}_{2}$ solid source. (d) $\mathrm{A} \mathrm{TiO}_{2}$ protection layer is grown by atomic layer deposition (ALD) on the top surface of the InP, and a Pt co-catalyst is evaporated onto the surface of the $\mathrm{TiO}_{2}$ to finish the device fabrication.

in the lateral direction and reducing loss of the metal source to evaporation. The resulting $\mathrm{InP}$ is polycrystalline with a thickness of $2-3 \mu \mathrm{m}$ (determined by the evaporated indium thin-film thickness) and a lateral grain size of up to $1 \mathrm{~mm} .^{22,23}$ This ultralarge grain size produces high optoelectronic performance, as demonstrated previously. ${ }^{22}$

To produce p-type InP for use as a photocathode, an ex situ surface diffusion doping process was carried out at a sample temperature of $425{ }^{\circ} \mathrm{C}$ using zinc phosphide $\left(\mathrm{Zn}_{3} \mathrm{P}_{2}\right)$ as the precursor (see the Experimental Methods section for details). $\mathrm{Zn}$ was chosen as the dopant because it is a commonly used acceptor in InP with a low activation energy. ${ }^{24}$ Notably, the $\mathrm{Zn}_{3} \mathrm{P}_{2}$ source was chosen to prevent InP decomposition by providing a phosphorus ambient present during the doping period. A $30 \mathrm{~nm}$ thick $n-\mathrm{TiO}_{2}$ protection layer was then deposited by atomic layer deposition (ALD) at $120{ }^{\circ} \mathrm{C}$ to protect the surface from corrosion during PEC measurements. ${ }^{12}$ Our previous studies ${ }^{13,25}$ have shown that this $n-\mathrm{TiO}_{2}$ layer on InP also provides a favorable surface energy band bending, allowing for transport of electrons while reflecting the holes due to the large barrier height at the $\mathrm{InP} / \mathrm{TiO}_{2}$ interface. Thus, higher $V_{\mathrm{OC}}$ was observed previously for $\mathrm{TiO}_{2}$-coated InP PV and PEC devices. ${ }^{13,25}$ The temperature of $\mathrm{TiO}_{2}$ deposition was optimized for device performance, as pictured in the $J-V$ comparison shown in Figure S1 (Supporting Information). Finally, Pt was used as an efficient catalyst material for water reduction. Throughout, growth and processing choices were influenced not only by the goal of cost reduction but also by stability and efficiency.

Morphological characterization of completed photocathodes is given by SEM images of a representative cross section in Figure $2 b, c$. Note that, as reported in our previous studies, each grain extends vertically from the Mo substrate to the top surface with a surface roughness defined primarily by the confinement of indium metal under the $\mathrm{SiO}_{2}$ cap, as demonstrated by the limited protrusions extending above large, high-quality grains shown in Figure $2 b^{22}$ The $30 \mathrm{~nm}$ thick, highly uniform, and conformal $\mathrm{TiO}_{2}$ layer depicted is designed to not only improve the protective qualities over the TF-VLS InP but also to act as an antireflective coating, improving the solar-weighted reflectance of the stack as

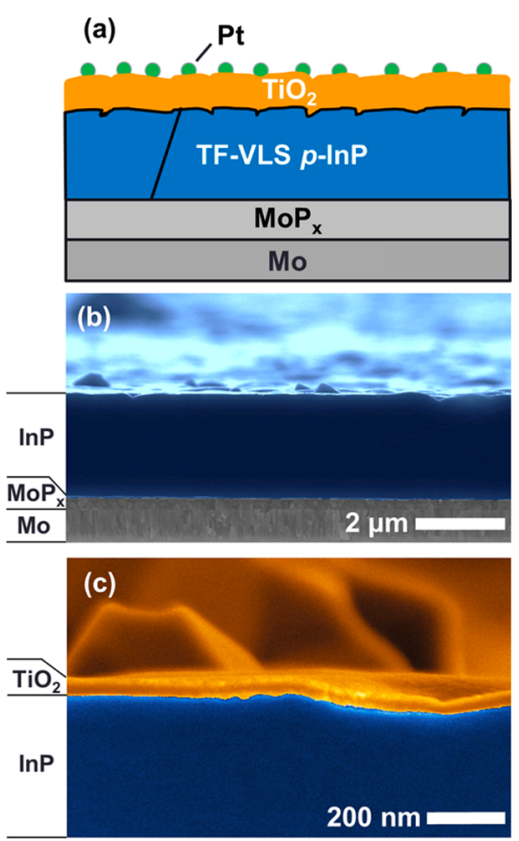

Figure 2. (a) Schematic of the typical device stack. (b) False-colored cross-sectional SEM image of the as-grown InP film. (c) Highmagnification SEM image (false-colored) of the $\mathrm{TiO}_{2}-\mathrm{InP}$ interface after $\mathrm{TiO}_{2}$ deposition by ALD.

compared to thinner coatings (Figure S2, Supporting Information). This structural and electrically functional antireflection coating is particularly important in a PEC cell because conventional multilayer antireflective coatings may not be possible due to the intimate semiconductor-solution contact necessary for charge transfer. It has also been shown previously that $\mathrm{TiO}_{2}$ thickness does not significantly impact charge transfer through the film; therefore, this design choice does not impact the electrical performance of our devices. ${ }^{10,25}$ Further optimization of the reflectance properties is possible with thicker $\mathrm{TiO}_{2}$ films, and sputtering of $\mathrm{TiO}_{2}$ as a protection layer and electron contact has been explored and implemented at much greater thicknesses in other photocathode systems. ${ }^{10}$ In the future, the electronic benefits and chemical stability shown in ALD $\mathrm{TiO}_{2}$ films could be combined with the increased throughput and lower cost of sputtering processes to yield optimal electronic, chemical passivation, and optical properties for photocathode cells.

The intrinsic optoelectronic and structural qualities of the material used in these PEC cells are also quite important, and these parameters were explored by steady-state micro-PL and $\mathrm{X}$-ray diffraction (XRD) measurements (Figure 3). XRD measurements (Figure 3a) indicate a high level of crystallinity of as-grown films, with peak positions closely matching a zinc blende powder reference 29 and with other peaks attributed to the $\mathrm{MoP}_{x} / \mathrm{Mo}$ substrate. ${ }^{30,31}$ As in our previous reports, a preferred (111) texture is observed for growth on Mo. In addition, while $\mathrm{Mo}$ is the most prominent feature of the substrate, $\mathrm{MoP}_{x}$ peaks are also visible in XRD (with $0.5<x<$ 0.8 ), demonstrating that there is some phosphorization of the Mo substrate during the growth process, as shown in previous explorations of InP growth. ${ }^{22,26,27}$ While not directly explored in this work, this modification of the Mo contact layer could have some impact on the device performance.

Steady-state, room-temperature PL data for Zn-doped TFVLS InP show peak positions, intensity, and full width half- 

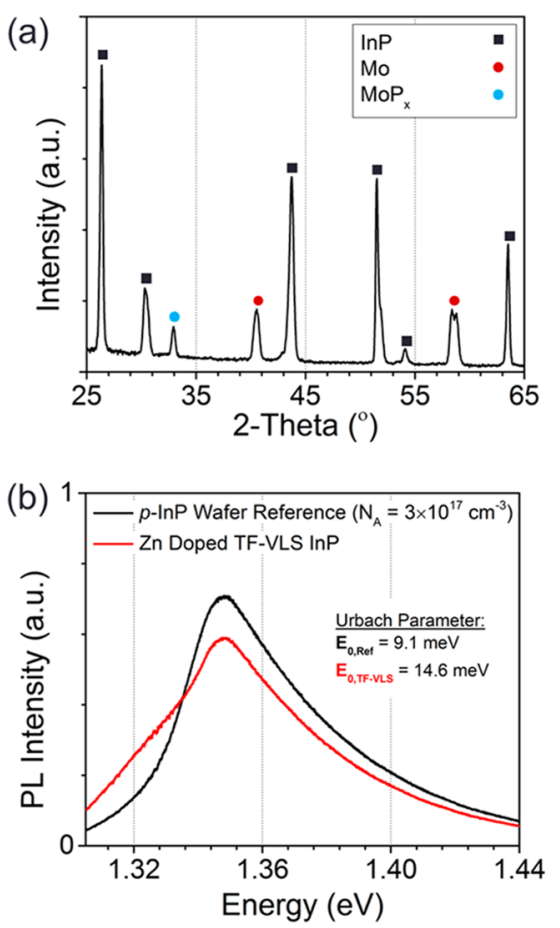

Figure 3. Physical and optical characterization of as-grown TF-VLS InP material and processed devices. (a) XRD data of as-grown material, indicating high crystallinity with narrow peaks closely matching a zinc blende powder reference. Peaks attributed to the growth substrate are also visible, here denoted as $\mathrm{Mo}$ and $\mathrm{Mo}_{1-x} \mathrm{P}_{x}$ $(0.5<x<0.8)$ according to a powder reference. (b) Steady-state micro-PL measurements, indicating similarity of intensity and shape to a p-type crystalline reference. The extracted Urbach tail parameter $E_{0}$ fitted from extracted absorbance (Supporting Information, Figure S3) is also reported for both the single-crystalline wafer reference and our p-type TF-VLS film. maxima close to those of a crystalline $p$-InP reference with similar doping concentration (Figure $3 \mathrm{~b}$ ), with only a lowenergy shoulder distinguishing the TF-VLS InP in this PL comparison. Particularly notable is the similarity in PL intensity and integrated counts as this indicates that our material approaches a similar luminescence yield and optically implied open-circuit voltage $\left(V_{\mathrm{OC}}\right)$ to the crystalline reference. ${ }^{22}$ The difference in peak shape can be attributed to the nonepitaxial growth and effects from the zinc doping process, a feature previously observed in impurity studies for InP and attributed to both the $\mathrm{Zn}$ impurity level and LO phonon coupling with the $\mathrm{Zn}$ impurity. ${ }^{28}$ To further characterize our material, we perform a fit of the Urbach tail using the RoosbroeckShockley $^{32}$ equation to extract a relative absorption parameter from our steady-state PL data. From a logarithm fit to the Urbach formula for band tail absorption $\left(\alpha(E)=\alpha_{0} \mathrm{e}^{\left[\left(E-E_{\text {ref }}\right) / E_{0}\right]}\right)$, we can extract the Urbach parameter $E_{0}$, which is a measure of band edge sharpness that can be correlated to disorder in a semiconductor. Details of this extraction can be found in the Supporting Information and in Figure S3. Comparing the single-crystal p-type wafer reference and our p-type TF-VLS film with a similar doping concentration, we see similar $E_{0}$ values of 9.1 and $14.6 \mathrm{meV}$, respectively, indicating band edge sharpness in our TF-VLS material in line with the single-crystal wafer. In summary, the PL data show that our TF-VLS material is of high optical quality for implementation in a device and that the growth and doping methods used here represent a promising route to polycrystalline thin-film material approaching equal performance to previously explored bulk single crystals.

Given the high optical and crystalline quality of our thin-film InP on Mo, we then sought characterization of the electronic properties and PEC performance of the complete photocathode cell. Before measuring our device in the final configuration, we
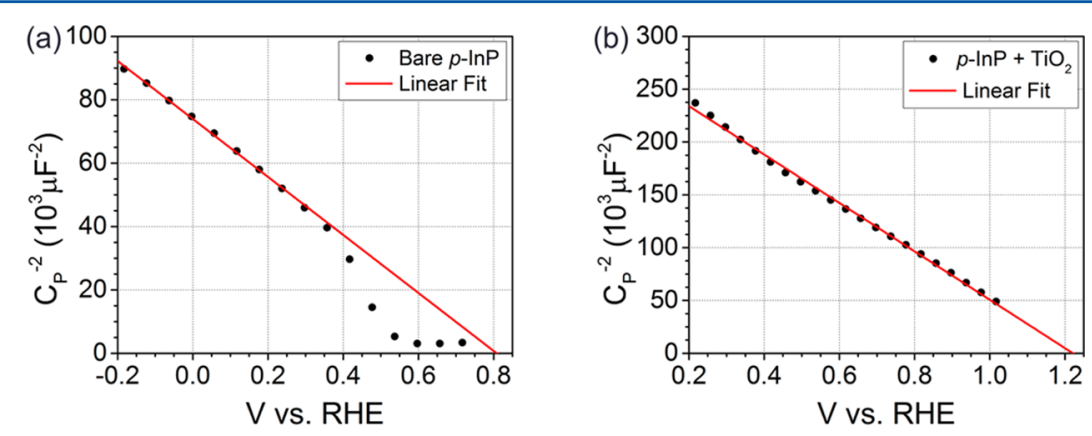

(c)
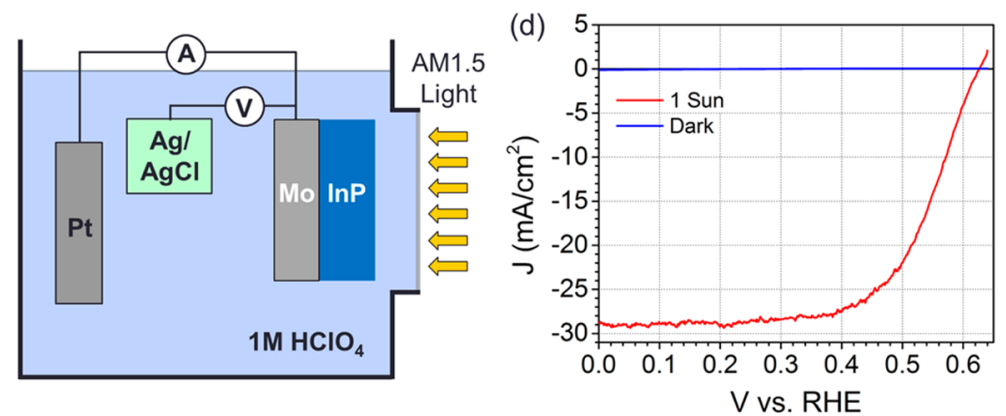

Figure 4. Mott-Schottky analysis and PEC performance of TF-VLS InP photocathodes with a $30 \mathrm{~nm} \mathrm{TiO}_{2}$ protection layer and Pt co-catalyst. (a,b) Mott-Schottky plot for p-doped TF-VLS before (a) and after (b) $\mathrm{TiO}_{2}$ deposition in a $\mathrm{pH} 7$ buffer. Linear fits are included for extraction of a flatband potential and doping concentration. (d) $J-V$ performance of a TF-VLS InP PEC device under AM1.5 illumination. $J-V$ test performed in 1 $\mathrm{M} \mathrm{HClO}_{4}$ with the cell depicted in (c). 
first aimed to understand the doping and solution-semiconductor electronic interface properties by performing MottSchottky capacitance-voltage measurements both without and with the protective $\mathrm{TiO}_{2}$ window layer (Figure 4a,b). Without the $\mathrm{TiO}_{2}$ layer, we can first measure the bare InP surface in order to extract the doping concentration and flatband potential $\left(V_{\mathrm{FB}}\right)$ of the InP-solution interface. The measured doping concentration of our material following the aforementioned doping process is $1.9 \times 10^{17} \mathrm{~cm}^{-3}$, a concentration in line with single-crystal wafers used in record photocathode cells. ${ }^{12}$ The $V_{\mathrm{FB}}$ extracted in this measurement is $0.81 \mathrm{~V}$ versus RHE, a value $\sim 0.55 \mathrm{eV}$ less than the InP band gap. As the InP conduction band is close to the $\mathrm{H}_{2} \mathrm{O} / \mathrm{H}_{2}$ redox potential at $\mathrm{pH}$ 0 according to previous reports, ${ }^{12,13}$ we would expect n-type surface inversion of the $p$-InP material at the liquid junction, a condition that would require a value of $V_{\mathrm{FB}}$ near the band gap to flatten the InP bands. The value of $V_{\mathrm{FB}}$ is thus a reflection of the surface band position prior to applying a potential as the built-in potential is applied to return the surface to the bulk band position. The lower value of $V_{\mathrm{FB}}$ despite an effective metal work function $\left(\Phi_{\mathrm{m}}\right)$ position near the InP conduction band edge suggests reduced sensitivity of the built-in potential to $\Phi_{\mathrm{m}}$, a condition reflective of Fermi-level pinning at the direct solution-InP interface. A similar result has been observed in previous explorations of the $p$-InP liquid junction (Figure 4a). ${ }^{33,34}$ Ideally, while the deposition of a $\mathrm{TiO}_{2}$ protective layer should primarily serve to allow electrode operation in strong acid solutions, the semiconductor heterojunction quality should also be maintained or improved following this process if it is a practical solution for high-efficiency cells because any degradation of the interface would lead to a direct reduction of obtainable $V_{\mathrm{OC}}{ }^{34}$ By measuring the flatband potential by the same method (Figure 4b), a measurement enabled due to rapid depletion of the thin $\mathrm{TiO}_{2}$ layer under reverse bias, we see that the $V_{\mathrm{FB}}$ of the $\mathrm{InP} / \mathrm{TiO}_{2}-$ solution interface shifts positively to $1.22 \mathrm{~V}$. This positive shift implies that indeed the $\mathrm{TiO}_{2}$ deposition improves the $\mathrm{InP}$ junction as the proximity of $V_{\mathrm{FB}}$, and thus the built-in potential, to the band gap energy places the redox potential far from the valence band, a condition that maximizes possible photovoltage in a solar device. ${ }^{34}$ As discussed in prior work on $\mathrm{InP}-\mathrm{TiO}_{2} \mathrm{p}-\mathrm{n}$ heterojunctions, we have created a thin-film cell with a high built-in potential, as well as a large barrier to minority holes that prevents interface recombination and supports charge separation. ${ }^{13,25}$ Therefore, the application of our $\mathrm{TiO}_{2}$ heterojunction contact provides both a chemically inert surface and an ideal structure for a highefficiency cathode cell.

Finally, TF-VLS InP photocathode device performance was evaluated directly on the basis of $J-V$ measurements in $1 \mathrm{M}$ $\mathrm{HClO}_{4}$ (Figure $4 \mathrm{~d}$ ). Light and dark $J-V$ sweeps were measured with a three-electrode configuration depicted in Figure 4c, under a simulated AM1.5 spectrum at $100 \mathrm{~mW} / \mathrm{cm}^{2}$, showing a short-circuit current density $\left(J_{\mathrm{SC}}\right)$ of $29.4 \mathrm{~mA} / \mathrm{cm}^{2}$ and a photocurrent onset of approximately $630 \mathrm{mV}$ versus RHE, with no significant features in the dark $J-V$ curve that would suggest unwanted electrochemical side reactions. Moreover, this performance level is consistent across multiple devices fabricated under these conditions, with a mean $V_{\mathrm{OC}}$ of 609 $\mathrm{mV}$ and mean $J_{\mathrm{SC}}$ of $27.4 \mathrm{~mA} / \mathrm{cm}^{2}$. More detailed device statistics are available in Figure S4 (Supporting Information). In addition, we have measured sustained photocurrent and $100 \%$ Faradaic efficiency for $\mathrm{H}_{2}$ over more than $2 \mathrm{~h}$, suggesting that our device structure is stable against corrosion during testing
(Figures S5 and S6, Supporting Information). Comparing device performance, single-crystalline bulk wafers have a $J_{\mathrm{SC}}$ of $25.5 \mathrm{~mA} / \mathrm{cm}^{2}$ and a photocurrent onset potential of approximately $810 \mathrm{mV}$ versus RHE. ${ }^{12}$ The lower $V_{\mathrm{OC}}$ for the TF-VLS InP compared to single-crystal wafers, despite the comparable high PL yields both as-grown ${ }^{22}$ and after doping (Figure 3), is attributed to a nonideal back contact and remaining observable crystal defects such as twin boundaries and grain boundaries. For the back contact, the effects of the $\mathrm{MoP}_{x}$ present below the InP are not known, and previous reports suggest that a $\mathrm{Zn}$-based metal alloy is necessary for ohmic contact to p-type InP. Therefore, further optimization in the doping process or growth substrate may improve photocathode performance. ${ }^{35,36}$ To this end, contact improvement strategies such as film transfer and alternate nucleation and contact layers for film growth are being pursued; ${ }^{22,23}$ therefore, significant improvement in overall photocathode performance using TF-VLS InP is certainly possible.

Nevertheless, the performance obtained here is still quite remarkable, with comparable voltage and improved efficiency compared to most non-III-V cathode technologies (Table 1).

Table 1. Tabulated Half-Cell STH Efficiencies, $V_{\text {OC }}$, and $J_{S C}$ of Reported Photocathode Devices

\begin{tabular}{|c|c|c|c|c|}
\hline system: absorber/catalyst & $\begin{array}{l}E_{\mathrm{G}} \\
(\mathrm{eV})\end{array}$ & $\begin{array}{c}V_{\text {OC }}(\mathrm{V} \text { vs } \\
\text { RHE })\end{array}$ & $\begin{array}{c}\left|J_{\mathrm{SC}}\right| \\
\left(\mathrm{mA} / \mathrm{cm}^{2}\right)\end{array}$ & $\eta(\%)$ \\
\hline Si microwire ${ }^{a}$ & 1.1 & 0.54 & 15 & 5.8 \\
\hline planar $\mathrm{Si}^{a}$ & 1.1 & 0.56 & 28 & 9.6 \\
\hline microtextured $\mathrm{Si}^{c}$ & 1.1 & 0.57 & 31.8 & 10.6 \\
\hline amorphous $\mathrm{Si}^{b}$ & 1.7 & 0.93 & 11.6 & 6 \\
\hline $\mathrm{WSe}_{2}$ crystal $^{d}$ & 1.2 & 0.63 & 24.5 & 7.2 \\
\hline $\mathrm{Cu}_{2} \mathrm{O} / \mathrm{MoS}_{2+x}{ }^{e}$ & 2.2 & 0.45 & 5.7 & 0.85 \\
\hline $\mathrm{CuGaSe} / \mathrm{Cd} S^{f}$ & 1.68 & 0.65 & 8.7 & 0.83 \\
\hline InP wafer nanopillars ${ }^{g}$ & 1.35 & 0.73 & 37 & 14 \\
\hline $\mathrm{InP}$ wafer/(Rh, H sat. $)^{h}$ & 1.35 & 0.64 & 28.6 & 13.3 \\
\hline InP wafer $/ \mathrm{TiO}_{2}{ }^{i}$ & 1.35 & 0.81 & 25.5 & 12.2 \\
\hline this work & 1.35 & 0.63 & 29.4 & 11.6 \\
\hline
\end{tabular}

(TF-VLS InP/ $\mathrm{TiO}_{2}$ )

${ }^{a}$ Reference 8. ${ }^{b}$ Reference 10. ${ }^{c}$ Reference $38 .{ }^{d}$ Reference 18. ${ }^{e}$ Reference 37. ${ }^{f}$ Reference $21 .{ }^{g}$ Reference $12 .{ }^{h}$ Reference $39,81.7$ $\mathrm{mW} / \mathrm{cm}^{2}$ illumination. ${ }^{i}$ Reference 13 .

As the water splitting PEC system requires large overpotentials beyond a minimum of $1.23 \mathrm{~V}$ for normal operation, the ultimate achievable voltage of a material is of utmost importance to its viability. When compared to single-crystal systems, the TF-VLS InP photocathode is favorable over silicon and $\mathrm{WSe}_{2}$ in voltage and possesses one of the highest shortcircuit current densities of all reported photocathodes with a competitive fill factor of $63 \%$. With efficiency closely approaching that of crystalline InP wafer devices, the TF-VLS InP photocathode is poised as a promising future choice in high-efficiency PEC systems.

In this work, we have demonstrated a stable, efficient photocathode device utilizing a low-cost, scalable fabrication technique that avoids expensive epitaxial growth processes and maintains high voltages and efficiencies necessary for viable operation of the water splitting PEC system. The devices presented in this work are the first demonstrated using this growth method, a technique that decreases usage of precious feedstock materials in high-efficiency III-V systems. While the existing performance is promising, the implied potential from this growth method, on the basis of measurements of the 
material's intrinsic quality, suggests that much more can be done to bring the system and structures explored in this work up to and beyond the crystalline wafers. To this end, the TFVLS growth method will be a valuable tool for production of higher-efficiency photocathodes, with precisely controlled nucleation and millimeter-scale grain sizes as the ultimate limit in highly scalable thin-film PEC technology.

\section{EXPERIMENTAL METHODS}

Fabrication Process. InP materials used in this work were grown using a recently developed TF-VLS growth method. ${ }^{22}$ Molybdenum foil substrates were degreased in acetone and isopropanol, followed by a hydrochloric acid etch and a deionized (DI) water rinse and nitrogen blow dry. The cleaned substrates were then loaded immediately into an electron beam evaporator, and $2 \mu \mathrm{m}$ of $99.9999 \%$ pure indium metal was deposited on the foils, followed by a $40 \mathrm{~nm} \mathrm{SiO}_{2}$ capping layer. After this, the coated substrates were placed in a tube furnace, where they were phosphorized according to a previously described method utilizing $10 \% \mathrm{PH}_{3}$ in $\mathrm{H}_{2}$ as the phosphorus source. $^{22}$ The typical growth process involves heating the substrates to $750{ }^{\circ} \mathrm{C}$ in $\mathrm{H}_{2}$ ambient, followed by a $20 \mathrm{~min}$ phosphorization step in $10 \mathrm{sccm}$ of $10 \% \mathrm{PH}_{3}$. This process yields an n-type InP layer approximately $3 \mu \mathrm{m}$ in thickness, after etching of the $\mathrm{SiO}_{2}$ capping layer in $49 \% \mathrm{HF}$. As we wish to extract minority electrons for the reduction of protons in solution, this n-type as-grown material must then be doped ptype. For this process, a closed-space sublimation system is used, with the TF-VLS InP placed on a $2 \mathrm{~mm}$ quartz spacer above $\mathrm{Zn}_{3} \mathrm{P}_{2}$ (99.999\%) powder used as the $\mathrm{Zn}$ acceptor source. The powder and substrate, held in thermal contact to graphite blocks, are heated separately by quartz lamps at 520 and $425{ }^{\circ} \mathrm{C}$, respectively, for a total doping time of $1 \mathrm{~h}$. The acceptor doping concentration in the InP layer using this method is approximately $10^{17} \mathrm{~cm}^{-3}$ as extracted by MottSchottky analysis. The doped samples are cleaned in five cycles of an acid treatment, utilizing $1 \% \mathrm{HCl}, 1 \% \mathrm{HNO}_{3}$, and DI water in succession to remove surface layers that may have been damaged during the doping step. The InP films are then coated with a $30 \mathrm{~nm}$ thick layer of titanium oxide $\left(\mathrm{TiO}_{2}\right)$ by an ALD process using titanium tetraisopropoxide and water and a deposition temperature of $120^{\circ} \mathrm{C}$. Finally, to enhance catalytic activity at the solution interface, a thin layer of platinum (thickness of approximately $1 \mathrm{~nm}$ ) co-catalyst is deposited by electron beam evaporation.

Physical Characterization. Scanning electron microscope (SEM) images were taken using a Zeiss Gemini Ultra-55. XRD data were taken using a Bruker AXS D8 Discover GADDS XRD diffractometer system. Steady-state micro-PL data were taken using a $633 \mathrm{~nm} \mathrm{HeNe}$ laser, and the detector was a silicon CCD.

PEC Characterization. PEC devices were characterized using a BioLogic SP-300 bipotentiostat in a three-electrode, quartzwindowed cell with a BASi RE5B $\mathrm{Ag} / \mathrm{AgCl}$ reference electrode, platinum wire counter electrode, and TF-VLS InP as the working electrode. The $1 \mathrm{M} \mathrm{HClO}_{4}$ was used as the electrolyte for $J-V$ experiments, and a $\mathrm{pH} 7$ buffer solution was used for Mott-Schottky measurements. The illumination source for $J-$ $V$ characterization, stability, and $\mathrm{H}_{2}$ generation measurements was a Solar Light 16S-300-005 solar simulator with AM1.5 filter. The light intensity of $100 \mathrm{~mW} / \mathrm{cm}^{2}$ was adjusted and calibrated using a Solar Light PMA-2100 radiometer and a PMA-2144 pyranometer, verified using a crystalline InP electrode IPCE calibrated against a NIST traceable photodiode (71648) from Newport. $\mathrm{H}_{2}$ generation data were measured using an Agilent 490 micro gas chromatograph (GC), with $\mathrm{N}_{2}$ carrier gas and a $0.1 \mathrm{M} \mathrm{H}_{2} \mathrm{SO}_{4}$ electrolyte to avoid corrosion of internal GC components.

\section{ASSOCIATED CONTENT}

\section{Supporting Information}

Effect of $\mathrm{TiO}_{2}$ deposition temperature on cell performance; calculated solar-weighted cell reflectance as a function of $\mathrm{TiO}_{2}$ thickness; Urbach tail analysis; PEC stability measurements; and $\mathrm{H}_{2}$ gas production Faradaic efficiency measurements. The Supporting Information is available free of charge on the ACS Publications website at DOI: 10.1021/acs.jpclett.5b00744.

\section{AUTHOR INFORMATION}

\section{Corresponding Author}

*E-mail: ajavey@eecs.berkeley.edu.

\section{Notes}

The authors declare no competing financial interest.

\section{ACKNOWLEDGMENTS}

This work was funded by the Bay Area Photovoltaics Consortium (BAPVC). Optical characterization was funded by the Director, Office of Science, Office of Basic Energy Sciences, Material Sciences and Engineering Division of the U.S. Department of Energy under Contract No. DE-AC0205CH11231. PEC device characterization was performed at the Joint Center for Artificial Photosynthesis, supported through the Office of Science of the U.S. Department of Energy under Award Number DE-SC0004993. Imaging and XRD were performed at The Molecular Foundry under Contract No. DE-AC02-05CH11231.

\section{REFERENCES}

(1) Grätzel, M. Photoelectrochemical Cells. Nature 2001, 414, 338344.

(2) Walter, M. G.; Warren, E. L.; McKone, J. R.; Boettcher, S. W.; Mi, Q.; Santori, E. A.; Lewis, N. S. Solar Water Splitting Cells. Chem. Rev. 2010, 110, 6446-6473.

(3) Turner, J. A. Sustainable Hydrogen Production. Science 2004, 305, 972-974.

(4) Seger, B.; Castelli, I. E.; Vesborg, P. C. K.; Jacobsen, K. W.; Hansen, O.; Chorkendorff, I. Energy Environ. Sci. 2014, 7, 2397.

(5) Maier, C. U.; Specht, M.; Bilger, G. Hydrogen Evolution on Platinum-Coated $p$-Silicon Photocathodes. Int. J. Hydrogen Energy 1996, 21, 859-864.

(6) Hou, Y.; Abrams, B. L.; Vesborg, P. C. K.; Björketun, M. E.; Herbst, K.; Bech, L.; Setti, A. M.; Damsgaard, C. D.; Pedersen, T.; Hansen, O.; Rossmeisl, J.; Dahl, S.; Nørskov, J. K.; Chorkendorff, I. Bioinspired Molecular Co-Catalysts Bonded to a Silicon Photocathode for Solar Hydrogen Evolution. Nat. Mater. 2011, 10, 434-438.

(7) Seger, B.; Pedersen, T.; Laursen, A. B.; Vesborg, P. C. K.; Hansen, O.; Chorkendorff, I. Using $\mathrm{TiO}_{2}$ as a Conductive Protective Layer for $\mathrm{H}_{2}$ Evolution. J. Am. Chem. Soc. 2013, 135, 1057-1064.

(8) Boettcher, S. W.; Warren, E. L.; Putnam, M. C.; Santori, E. A.; Turner-Evans, D.; Kelzenberg, M. D.; Walter, M. G.; McKone, J. R.; Brunschwig, B. S.; Atwater, H. A.; Lewis, N. S. Photoelectrochemical Hydrogen Evolution Using Si Microwire Arrays. J. Am. Chem. Soc. 2011, 133, 1216-1219.

(9) Oh, I.; Kye, J.; Hwang, S. Enhanced Photoelectrochemical Hydrogen Production from Silicon Nanowire Array Photocathode. Nano Lett. 2012, 12, 298-302.

(10) Lin, Y.; Battaglia, C.; Boccard, M.; Hettick, M.; Yu, Z.; Ballif, C.; Ager, J. W.; Javey, A. Amorphous Si Thin Film Based Photocathodes 
with High Photovoltage for Efficient Hydrogen Production. Nano Lett. 2013, 13 (11), 5615-5618.

(11) Szklarczyk, M.; Bockris, J. O. Photoelectrochemical Evolution of Hydrogen on $p$-Indium Phosphide. J. Phys. Chem. 1984, 88, 52415245.

(12) Lee, M. H.; Takei, K.; Zhang, J.; Kapadia, R.; Zheng, M.; Chen, Y.-Z.; Nah, J.; Matthews, T. S.; Chueh, Y.-L.; Ager, J. W.; Javey, A. pType InP Nanopillar Photocathodes for Efficient Solar-Driven Hydrogen Production. Angew. Chem., Int. Ed. 2012, 51, 10760-10764.

(13) Lin, Y.; Kapadia, R.; Yang, J.; Zheng, M.; Chen, K.; Hettick, M.; Yin, X.; Battaglia, C.; Sharp, I. D.; Ager, J. W.; Javey, A. The Role of $\mathrm{TiO}_{2}$ Surface Passivation on Improving the Performance of $p$-InP Photocathodes. J. Phys. Chem. C 2015, 119, 2308-2313.

(14) Heller, A.; Miller, B.; Lewerenz, H. J.; Bachmann, K. J. An Efficient Photocathode for Semiconductor Liquid Junction Cells: 9.4\% Solar Conversion Efficiency with $p$ - InP/ $/ \mathrm{VCl}_{3}-\mathrm{VCl}_{2}-\mathrm{HCl} / \mathrm{C}$. J. Am. Chem. Soc. 1980, 102, 6555-6556.

(15) Heller, A. Hydrogen Evolving Solar Cells. Catal. Rev.: Sci. Eng. 2013, 26, 655-681.

(16) Schulte, K.; Lewerenz, H. Combined Photoelectrochemical Conditioning and Photoelectron Spectroscopy Analysis of InP Photocathodes. I. The Modification Procedure. Electrochim. Acta 2002, 47, 2633-2638.

(17) Arai, T.; Sato, S.; Uemura, K.; Morikawa, T.; Kajino, T.; Motohiro, T. Photoelectrochemical Reduction of $\mathrm{CO}_{2}$ in Water under Visible-Light Irradiation by a $p$-Type InP Photocathode Modified with an Electropolymerized Ruthenium Complex. Chem. Commun. 2010, 46, 6944-6946.

(18) McKone, J. R.; Pieterick, A. P.; Gray, H. B.; Lewis, N. S. Hydrogen Evolution from $\mathrm{Pt} / \mathrm{Ru}$-Coated $\mathrm{p}$-Type $\mathrm{WSe}_{2}$ Photocathodes. J. Am. Chem. Soc. 2013, 135, 223-231.

(19) Siripala, W.; Ivanovskaya, A.; Jaramillo, T. F.; Baeck, S.-H.; McFarland, E. W. A $\mathrm{Cu}_{2} \mathrm{O} / \mathrm{TiO}_{2}$ Heterojunction Thin Film Cathode for Photoelectrocatalysis. Sol. Energy Mater. Sol. Cells 2003, 77, 229237.

(20) Paracchino, A.; Laporte, V.; Sivula, K.; Grätzel, M.; Thimsen, E. Highly Active Oxide Photocathode for Photoelectrochemical Water Reduction. Nat. Mater. 2011, 10, 456-461.

(21) Moriya, M.; Minegishi, T.; Kumagai, H.; Katayama, M.; Kubota, J.; Domen, K. Stable Hydrogen Evolution from CdS-Modified $\mathrm{CuGaSe}_{2}$ Photoelectrode under Visible-Light Irradiation. J. Am. Chem. Soc. 2013, 135, 3733-3735.

(22) Kapadia, R; Yu, Z.; Wang, H.-H. H.; Zheng, M.; Battaglia, C.; Hettick, M.; Kiriya, D.; Takei, K.; Lobaccaro, P.; Beeman, J. W.; Ager, J. W.; Maboudian, R.; Chrzan, D. C.; Javey, A. A Direct Thin-Film Path Towards Low-Cost Large-Area III-V Photovoltaics. Sci. Rep. 2013, 3, 2275.

(23) Kapadia, R.; Yu, Z.; Hettick, M.; Xu, J.; Zheng, M. S.; Chen, C.Y.; Balan, A. D.; Chrzan, D. C.; Javey, A. Deterministic Nucleation of InP on Metal Foils with the Thin-Film Vapor-Liquid-Solid Growth Mode. Chem. Mater. 2014, 26 (3), 1340-1344.

(24) Yang, J. J.; Ruth, R. P.; Manasevit, H. M. Electrical Properties of Epitaxial Indium Phosphide Films Grown by Metalorganic Chemical Vapor Deposition. J. Appl. Phys. 1981, 52, 6729.

(25) Yin, X.; Battaglia, C.; Lin, Y.; Chen, K.; Hettick, M.; Zheng, M.; Chen, C.-Y.; Kiriya, D.; Javey, A. $19.2 \%$ Efficient InP Heterojunction Solar Cell with Electron Selective $\mathrm{TiO}_{2}$ Contact. ACS Photonics 2014, 1 (12), 1245-1250.

(26) Zheng, M.; Yu, Z.; Joon Seok, T.; Chen, Y.-Z.; Kapadia, R; Takei, K.; Aloni, S.; Ager, J. W.; Wu, M.; Chueh, Y.-L.; Javey, A. High Optical Quality Polycrystalline Indium Phosphide Grown on Metal Substrates by Metalorganic Chemical Vapor Deposition. J. Appl. Phys. 2012, 111, 123112.

(27) Kiriya, D.; Zheng, M.; Kapadia, R.; Zhang, J.; Hettick, M.; Yu, Z.; Takei, K.; Hank Wang, H.-H.; Lobaccaro, P.; Javey, A. Morphological and Spatial Control of InP Growth Using ClosedSpace Sublimation. J. Appl. Phys. 2012, 112, 123102.

(28) Williams, E. W.; Elder, W.; Astles, M. G.; Webb, M.; Mullin, J. B.; Straughan, B.; Tufton, P. J. Indium Phosphide I. A Photo- luminescence Materials Study. J. Electrochem. Soc. 1973, 120 (12), $1741-1749$.

(29) ICDD PDF-2, Entry 00-032-0452; The International Centre for Diffraction Data: Newtown Square, PA, 2003.

(30) ICDD PDF-2, Entry 00-065-6024; The International Centre for Diffraction Data: Newtown Square, PA, 2003.

(31) ICDD PDF-2, Entry 00-089-5156; The International Centre for Diffraction Data: Newtown Square, PA, 2003.

(32) Kost, A.; Lee, H. C.; Zou, Y.; Dapkus, P. D.; Garmire, E. BandEdge Absorption Coefficients from Photoluminescence in Semiconductor Multiple Quantum Wells. Appl. Phys. Lett. 1989, 54, 13561358.

(33) Heller, A. Conversion of Sunlight into Electrical Power and Photoassisted Electrolysis of Water in Photoelectrochemical Cells. Acc. Chem. Res. 1981, 14 (5), 154-162.

(34) Bard, A. J.; Bocarsly, A. B.; Fan, F. R. F.; Walton, E. G.; Wrighton, M. S. The Concept of Fermi Level Pinning at Semiconductor/Liquid Junctions. Consequences for Energy Conversion Efficiency and Selection of Useful Solution Redox Couples in Solar Devices. J. Am. Chem. Soc. 1980, 102 (11), 3671-3677.

(35) Piotrowska, A.; Guivarc'h, A.; Pelous, G. Ohmic Contacts to III-V Compound Semiconductors: A Review of Fabrication Techniques. Solid-State Electron. 1983, 26, 179-197.

(36) Park, M.-H.; Wang, L. C.; Cheng, J. Y.; Palmstrom, C. J. Low Resistance Ohmic Contact Scheme $\left(\sim \mu \Omega \mathrm{cm}^{2}\right)$ to $p$-InP. Appl. Phys. Lett. 1997, 70, 99-101.

(37) Morales-Guio, C. G.; Tilley, S. D.; Vrubel, H.; Grätzel, M.; Hu, X. Hydrogen Evolution from a Copper(I) Oxide Photocathode Coated with an Amorphous Molybdenum Sulphide Catalyst. Nat. Commun. 2014, 5, 3059.

(38) Kast, M. G.; Enman, L. J.; Gurnon, N. J.; Nadarajah, A.; Boettcher, S. W. Solution-Deposited $\mathrm{F}: \mathrm{SnO}_{2} / \mathrm{TiO}_{2}$ as a Base-Stable Protective Layer and Antireflective Coating for Microtextured BuriedJunction $\mathrm{H}_{2}$-Evolving Si Photocathodes. ACS Appl. Mater. Interfaces 2014, 6 (24), 22830-22837.

(39) Heller, A. Hydrogen-Evolving Solar Cells. Science 1984, 223 (4641), 1141-1148. 